\title{
ZARISKI $F$-DECOMPOSITION AND LAGRANGIAN FIBRATION ON HYPERKÄHLER MANIFOLDS
}

\author{
Daisuke Matsushita And De-Qi Zhang
}

\begin{abstract}
For a compact hyperkähler manifold $X$, we show certain Zariski decomposition for every pseudo-effective $\mathbb{R}$-divisor. We also prove that any sequence of $D$-flops between projective hyperkähler manifolds terminates after finitely many steps.
\end{abstract}

\section{Introduction}

It is a classical result that a pseudo-effective divisor on a compact complex surface has a Zariski decomposition. When $X$ is a compact complex manifold of dimension $>2$ and $D$ a pseudo-effective $\mathbb{R}$-divisor on $X$, we may consider two types of Zariskidecompositions: either in the sense of Fujita or in the sense of Cutkosky-KawamataMoriwaki as defined before Theorem 3.1. In general, such decompositions may not exist. On the other hand, there are also positive results for big divisors or for toric varieties; see [10, Remark 7-3-6, Th. 7-3-7] and [14, Chapter II, Remark 1.17].

We show the existence of such decompositions on a projective hyperkähler manifold (cf. the definition in 2.1).

Below is our first main theorem, a special case of the more elaborated Theorem 3.1.

Theorem 1.1. Let $X$ be a projective hyperkähler manifold and $D$ a pseudo-effective $\mathbb{R}$-divisor on $X$. Then there are a birational map $\sigma_{1}: X_{1} \rightarrow X$ from a projective hyperkähler manifold $X_{1}$ and a birational morphism $\sigma_{2}: X_{2} \rightarrow X$ from a projective manifold $X_{2}$ such that, for each $k \in\{1,2\}, D_{k}:=\sigma_{k}^{*} D$ has a Zariski-Fujita decomposition

$$
D_{k}=P_{k}+N_{k}
$$

in the sense of Fujita [7]. Namely, we have:

(i) the divisor $P_{k}$ is nef, i.e., $P_{k} \in \bar{K}(X)$, the closure of the Kähler cone $K(X)$; and

(ii) the divisor $N_{k}$ is effective; $F \geq \tau^{*} N_{k}$ holds whenever there are a birational morphism $\tau: X^{\prime} \rightarrow X_{k}$ and divisor $F \geq 0$ with $\tau^{*} D_{k}-F$ nef.

We remark that the decomposition $D_{k}=P_{k}+N_{k}(k=1,2)$ in Theorem 1.1 is also in the sense of Cutkosky-Kawamata-Moriwaki; see [10, Def. 7-3-2, 7-3-5] or the paragraph before Theorem 3.1 for the definition.

Received by the editors May 20, 2011.

2000 Mathematics Subject Classification. 14J40, 32Q15, 14E30, 53C26.

Key words and phrases. hyperkähler manifold, Zariski decomposition, Lagrangian fibration, termination of flops. 
Our next main theorem is used in the implication "Theorem $3.1 \Rightarrow$ Theorem 1.1", and is a consequence of Theorem 4.1: the termination of flops between projective hyperkähler manifolds.

Theorem 1.2. Let $X$ be a projective hyperkähler manifold, and $P$ an effective $\mathbb{R}$ divisor in the closed movable cone $\overline{\operatorname{Mov}}(X)$ (cf. the definition in 2.1). Then there is a birational map $\tau: X^{\prime} \rightarrow X$ from a projective hyperkähler manifold $X^{\prime}$ such that $\tau^{*} P$ is nef.

\section{Preliminary results}

\subsection{Conventions and terminology}

We use the conventions in $[8,11]$ and Hartshorne's book.

Let $X$ be a compact complex Kähler manifold. For an $\mathbb{R}$-divisor $\Delta$ on $X$, we set $H^{0}(X, \Delta):=H^{0}(X,\lfloor\Delta\rfloor)$, with $\lfloor\Delta\rfloor$ the integral part (or round down) of $\Delta$.

Set $H^{1,1}(X, \mathbb{R}):=H^{1,1}(X, \mathbb{C}) \cap H^{2}(X, \mathbb{R})$. The (closed) nef cone $\bar{K}(X)$ in $H^{1,1}(X, \mathbb{R})$ is the closure of the Kähler cone $K(X)$ of $X$. An element in $\bar{K}(X)$ is called a nef class. Let $\mathrm{NS}(X)$ be the Néron-Severi group. Set $\mathrm{NS}_{\mathbb{Q}}(X):=\mathrm{NS}(X) \otimes_{\mathbb{Z}} \mathbb{Q}$ and $\mathrm{N}^{1}(X):=\mathrm{NS}(X) \otimes_{\mathbb{Z}} \mathbb{R}$. We have $\mathrm{N}^{1}(X) \subseteq H^{1,1}(X, \mathbb{R})$.

The (closed) pseudo-effective divisor cone $\mathrm{PE}(X)$ in $\mathrm{N}^{1}(X)$ is the closure of effective $\mathbb{R}$-divisor classes on $X$. The closed movable cone $\overline{\operatorname{Mov}}(X)$ in $\mathrm{N}^{1}(X)$ is generated by the classes of fixed-component free divisors. We have $\overline{\operatorname{Mov}}(X) \subseteq \mathrm{PE}(X)$.

We now briefly recall some definitions related to hyperkähler manifolds, and refer to $[8$, pages $171,176,182-184,223-224]$ for details. The compact complex Kähler manifold $X$ is called hyperkähler (or irreducible holomorphic symplectic) if it is simply connected such that $H^{0}\left(X, \Omega_{X}^{2}\right)$ is spanned by an everywhere non-degenerate twoform $\sigma$. It follows then $\operatorname{dim} X=2 n$ for some integer $n \geq 1$. We normalize $\sigma$ so that $\int_{X}(\sigma \bar{\sigma})^{n}=1$.

There exists a primitive integral quadratic form $q_{X}(*)$ on $H^{2}(X, \mathbb{Z})$, the BeauvilleBogomolov form. Indeed, there is a positive constant $a$ such that

$$
q(L)=a \int_{X} L^{2}(\sigma \bar{\sigma})^{n-1}, \quad L \in H^{1,1}(X, \mathbb{C}) .
$$

This $q_{X}(*)$ is non-degenerate of signature $\left(3, b_{2}(X)-3\right)$. There is a so called BeauvilleFujiki number $c>0$ such that

$$
q(L)^{n}=c L^{2 n}, \quad L \in H^{2}(X, \mathbb{C}) .
$$

The positive cone $C(X)$ in $H^{1,1}(X, \mathbb{R})$ is the connected component of the open cone $\left\{\alpha \in H^{1,1}(X, R) \mid q(\alpha)>0\right\}$ that contains a Kähler class of $X$. The closure of $C(X)$ in $H^{1,1}(X, \mathbb{R})$ is denoted by $\bar{C}(X)$. The birational Kähler cone $\mathrm{BK}(\mathrm{X})$ in $H^{1,1}(X, \mathbb{R})$ is

$$
\operatorname{BK}(X)=\cup_{f: X \rightarrow X^{\prime}} f^{*} K\left(X^{\prime}\right)
$$

where $f: X \rightarrow X^{\prime}$ runs through all bimeromorphic maps $X \rightarrow X^{\prime}$ from $X$ to another compact hyperkähler manifold $X^{\prime}$. The closure of $\operatorname{BK}(X)$ in $H^{1,1}(X, \mathbb{R})$ is denoted by $\overline{\mathrm{BK}}(X)$. It is known that $\mathrm{BK}(X) \subseteq C(X)$ and hence $\overline{\mathrm{BK}}(X) \subseteq \bar{C}(X)$.

For our compact hyperkähler $X$, it is known that $\overline{\operatorname{Mov}}(X)=\overline{\mathrm{BK}}(X) \cap \mathrm{N}^{1}(X)$; see also [14, Chapter III, Def. 1.13 and 3.9] and Remark 3.3 (3) below. Further, $\overline{\mathrm{BK}}(X)$ coincides with Boucksom's modified nef cone $\mathrm{MN}(X)$ (cf. [4, Prop. 4.4 and Lemma 4.9] and [8, Prop. 28.7]). 
Lemma 2.2. Let $X$ be a compact hyperkähler manifold with $q(*)$ the primitive Beauville-Bogomolov quadratic form (and $q(*, *)$ its bilinear form) on $H^{2}(X, \mathbb{Z})$. Then we have:

(1) The birational Kähler cone $\overline{\mathrm{BK}}(X)$ is the intersection of $\bar{C}(X)$ and the dual of the pseudo-effective divisor (closed) cone $\mathrm{PE}(X) \subset H^{1,1}(X, \mathbb{R})$ with respect to $q(*, *)$. If $D \in \mathrm{PE}(X)$ and $q(D, E) \geq 0$ for every prime divisor $E$, then $D \in \overline{\mathrm{BK}}(X)$.

(2) If $D_{1}, D_{2}$ are distinct prime divisors, then $q\left(D_{1}, D_{2}\right) \geq 0$. Similarly, $q\left(D_{1}, D_{3}\right) \geq 0$ when $D_{3} \mid D_{1}$ is a pseudo-effective divisor on $D_{1}$.

(3) Suppose that $E_{i}$ are prime divisors with negative definite matrix $\left(q\left(E_{i}, E_{j}\right)\right)_{i, j}$. Then $E_{i}$ are linearly independent in the Neron-Severi group $\mathrm{NS}_{\mathbb{Q}}(X)$, and the Iitaka $D$-dimension $\kappa\left(X, \sum E_{i}\right)=0$. If $D \in \mathrm{PE}(X)$ and $E=\sum e_{i} E_{i}$ such that $q\left(D-E, E_{j}\right) \leq 0$ for all $j$, then $D-E \in \mathrm{PE}(X)$.

(4) $L \in H^{1,1}(X, \mathbb{R})$ belongs to $C(X)$ if and only if $q(L)>0$ and $q(L, \omega)>0$ for some Kähler class $\omega$.

(5) If an effective $\mathbb{Q}$-divisor $L \in \mathrm{NS}_{\mathbb{Q}}(X)$ satisfies $q(L)>0$, then $X$ is projective, and $L$ is big, i.e., $L=A+E$ for an ample $\mathbb{Q}$-divisor $A$ and an effective $\mathbb{Q}$ divisor $E$; further, $|s L|=|M|+F$ for some integer $s \geq 1$, with $M$ big, $|M|$ the movable part, and $F$ the fixed part.

(6) If $\sigma: X^{\prime} \rightarrow X$ is a bimeromorphic map from a compact hyperkähler manifold $X^{\prime}$, then it is isomorphic in codimenion one. Hence $\sigma^{*}$ is well defined on $H^{2}(X, \mathbb{C})$ and compatible with its Hodge structure, the Beauville Bogomolov quadratic form $q(*)$ and the birational Kähler cone.

(7) If $0 \neq L \in \bar{C}(X)$ and $D \in \operatorname{PE}(X)$ satisfy $q(L, D)=0$, then either $D$ and $L$ are parallel in $H^{1,1}(X, \mathbb{R})$ and $q(D)=0$, or $q(D)<0$.

(8) If $L_{1} \equiv L_{2}$ (numerical equivalence) for two $\mathbb{Q}$-divisors $L_{i}$, then $L_{1} \sim_{\mathbb{Q}} L_{2}$.

Proof. For (1), see [8, Prop. 28.7]. Note that $q(D) \geq 0$ in the second part.

For (2), since $\sigma \bar{\sigma} \mid D_{1} \cap D_{2}$ is weakly positive, one has

$$
q\left(D_{1}, D_{2}\right)=a \int_{D_{1} \cap D_{2}}\left(\sigma \bar{\sigma} \mid D_{1} \cap D_{2}\right)^{n-1} \geq 0 .
$$

For the first part of (3), suppose $E^{\prime}:=\sum a_{i} E_{i} \equiv \sum b_{j} E_{j}=: E^{\prime \prime}$ (numerical equivalence) for some $a_{i} \geq 0, b_{j} \geq 0$ and $E_{i} \neq E_{j}$. Then $0 \geq q\left(E^{\prime}\right)=q\left(E^{\prime}, E^{\prime \prime}\right) \geq 0$ by (2), and hence $E^{\prime}=0=E^{\prime \prime}$ by the negative-definite assumption. For the second part, write $D=\lim _{t \rightarrow \infty} D(t)$ and $D(t)=\sum d(t)_{i} E_{i}+D(t)^{\prime}$ with $d(t)_{i} \geq 0$, where $D(t)^{\prime}$ is an effective divisor and contains no any $E_{i}$. Since $D(t)$ has a limit and intersecting with a power of a Kähler class, we see that $d(t)_{i}$ are bounded and we let $\lim _{t \rightarrow \infty} d(t)_{i}=d_{i} \geq 0$. Then

$$
\begin{aligned}
0 & \geq q\left(D-E, E_{j}\right)=\lim _{t \rightarrow \infty} q\left(D(t)-E, E_{j}\right) \\
& \geq \lim _{t \rightarrow \infty} q\left(\sum\left(d(t)_{i}-e_{i}\right) E_{i}, E_{j}\right)=q\left(\sum\left(d_{i}-e_{i}\right) E_{i}, E_{j}\right) .
\end{aligned}
$$

Hence $\sum\left(d_{i}-e_{i}\right) E_{i} \geq 0$ by Zariski's lemma as in [12, Lemma 3.2]. Thus

$$
D-E=\lim _{t \rightarrow \infty}(D(t)-E)=\sum\left(d_{i}-e_{i}\right) E_{i}+\lim _{t \rightarrow \infty} D(t)^{\prime} \in \operatorname{PE}(X) .
$$


(4) is due to the very definition of the positive cone $C(X)$ (containing the Kähler cone $K(X)$ ).

The first part of (5) follows from [8, Prop. 26.13] and the claim in its proof, while the second part follows from the first part.

(6) is proved in [8, Prop. 21.6 and 25.14] (cf. (1)).

For $(7)$, see $\left[1\right.$, Chapter IV, (7.2)]) and note: $q(*)$ has signature $\left(1, b_{2}(X)-3\right)$ on $H^{1,1}(X, \mathbb{R})$.

(8) is true because the irregularity $h^{1}\left(X, \mathcal{O}_{X}\right)=0$.

\section{Proof of Theorems and their consequences}

In this section, we prove the results in the introduction as well as Theorem 3.1. Theorem 3.1 implies Theorem 1.1, thanks to Theorem 1.2.

The part (I) below has been given an analytic proof by Boucksom [4, Section 4]. The result in [4] is broader since Boucksom decomposes every pseudo-effective classes in $H^{1,1}(X, \mathbb{R})$ (which may not be a divisor class) as the sum of a modified nef class and the class of an effective exceptional divisor. The purpose of including the Part (I) here is to stress that it has also an algebraic constructive proof; to be precise, we will see that it follows also from the original Zariski's lemmas [17] as in Fujita [6] for surfaces (cf. also [12, Chapter I, Section 3.1-3.7]).

The part (II) below shows, under certain condition $(*)$ (always true for projective $X$ by Theorem 1.2), the existence of the Zariski decomposition in the sense of Fujita [7], i.e., requiring (II) (i-ii) in Theorem 3.1 (II); and hence is also the Zariski decomposition in the sense of Cutkosky-Kawamata-Moriwaki, i.e., requiring (i), (iii) in Theorem 3.1 (II) and (ii)': $N_{k}$ is effective.

Curious readers may try to extend Theorem 3.1 (II) to deal with the modified nef classes, by using the characterization of Demailly-Paun for nef classes.

Theorem 3.1. Let $X$ be a compact hyperkähler manifold and $D \in \mathrm{PE}(X)$ a pseudoeffective divisor. Then we have:

(I) There is the following Zariski q-decomposition $D=P_{D}+N_{D}$ such that:

(i) the divisor $P_{D} \in \overline{\mathrm{BK}}(X)$;

(ii) the divisor $N_{D} \geq 0$; either $N_{D}=0$, or for $\operatorname{Supp} N_{D}=\cup N_{i}$, the Beauville-Bogomolov quadratic matrix $\left(q\left(N_{i}, N_{j}\right)\right)_{i, j}$ is negative definite; and

(iii) $q\left(P_{D}, N_{i}\right)=0$ for all $i$.

The above Zariski q-decomposition is unique. Moreover, if $D \geq 0$ then $P_{D} \geq 0$; if $D$ is a $\mathbb{Q}$-divisor then so are $P_{D}$ and $N_{D}$. Finally, for all integers $s \geq 1$, we have the natural isomorphism: $H^{0}\left(X, s P_{D}\right) \cong H^{0}(X, s D)$.

(II) Suppose the condition (*) that there is a bimeromorphic map $\sigma_{1}: X_{1} \rightarrow X$ from a compact hyperkähler manifold $X_{1}$ such that $\sigma_{1}^{*} P_{D}$ is nef (i.e., $\sigma_{1}^{*} P_{D} \in$ $\bar{K}\left(X_{1}\right)$; see Theorem 1.2). Then there is a bimeromorphic morphism $\sigma_{2}$ : $X_{2} \rightarrow X$ such that for each $k \in\{1,2\}$ there is a Zariski-Fujita decomposition (or Zariski F-decomposition for short) $D_{k}=P_{k}+N_{k}$ for $D_{k}:=\sigma_{k}^{*} D$, in the sense of Fujita [7] (cf. [10, Def. 7-3-2, 7-3-5]). Thus the following hold.

(i) the divisor $P_{k}$ is nef, i.e., $P_{k} \in \bar{K}(X)$, the nef cone;

(ii) the divisor $N_{k}$ is effective; $F \geq \tau^{*} N_{k}$ holds whenever there are a bimeromorphic morphism $\tau: X^{\prime} \rightarrow X_{k}$ and divisor $F \geq 0$ with $\tau^{*} D_{k}-F$ nef; 
(iii) the natural isomorphism: $H^{0}\left(X_{k}, s P_{k}\right) \cong H^{0}\left(X_{k}, s D_{k}\right)$, for all integers $s \geq 1$.

The above Zariski F-decomposition is unique. (iii) follows from (i) and (ii). Moreover, if $D \geq 0$ then $P_{k} \geq 0$; if $D$ is a $\mathbb{Q}$-divisor then so are $P_{k}$ and $N_{k}$.

The condition $(*)$ in Theorem 3.1 is always satisfied by projective $X$, by Theorem 1.2.

The result below is parallel to the surface case.

Corollary 3.2. With the notation in Theorem $3.1(\mathrm{I})$, the Iitaka D-dimension $\kappa(X, D)$ equals $\kappa\left(X, P_{D}\right)$; we have $\kappa(X, D)=\operatorname{dim} X$ (maximal case) if and only if $q\left(P_{D}\right)>0$.

Remark 3.3. (1) The Zariski decomposition $D_{k}=P_{k}+N_{k}$ in Theorem 3.1 (II) is also in the sense of Cutkosky-Kawamata-Moriwaki (cf. [10, Def. 7-3-2, 7-3-5]).

(2) By the proof, the Zariski-Fujita decomposition in Theorem 3.1 (II) for $D_{1}$ on $X_{1}$, coincides with the Zariski q-decomposition in Theorem 3.1 (I) for $D_{1}$.

(3) The $N_{D}$ in Theorem 3.1 (I) is the smallest effective divisor such that $D-N_{D} \in$ $\overline{\mathrm{BK}}(X)$ : if $N^{\prime} \geq 0$ such that $P^{\prime}:=D-N^{\prime} \in \overline{\mathrm{BK}}(X)$, then $N^{\prime} \geq N_{D}$. Indeed, $q\left(N^{\prime}-N_{D}, N_{i}\right)=q\left(P_{D}-P^{\prime}, N_{i}\right)=-q\left(P^{\prime}, N_{i}\right) \leq 0$ (cf. Lemma 2.2 (1)) for every $N_{i} \leq N_{D}$ and hence $N^{\prime}-N_{D} \geq 0$ by Zariski's lemma as in the proof of [12, Chapter I, 3.2].

(4) By the above reasoning and Lemma 2.2 (2), the Zariski $q$-decomposition in Theorem 3.1(I) (for projective $X$ ) coincides with both the $\sigma$ - and $\nu$ decomposition in Nakayama [14, Chapter III, Def. 1.16 and 3.2].

\subsection{Proof of Theorem 3.1}

Part (I) is proved in [4, Section 4], but the intersection-form based arguments in Zariski [17] and Fujita [6] (cf. also Miyanishi [12, Chapter I, 3.1-3.7]) work well for (I) with almost no change, although we use $q(*)$ and Lemma 2.2 instead of the intersection form for surfaces. Nef divisors on a surface correspond to elements in the birational Kähler cone $\overline{\mathrm{BK}}(X)$ (cf. Lemma $2.2(1)$ ). The only non-intersection based usage of the Riemann-Roch theorem in [12, Lemma 3.5] may be replaced by Lemma 2.2 (5) (3).

The sketch of a constructive proof for (I): Let $E_{i}\left(1 \leq i \leq t_{1}\right)$ be all prime divisors such that $q\left(D, E_{i}\right)<0$. Then $\left(q\left(E_{i}, E_{j}\right)\right)_{i, j}$ is a negative definite matrix [ibid. Proof of 3.6]. Let $F_{1}$ be a (non-negative, by Zariski's lemma) combination of $E_{i}$ such that $D_{1}:=D-F_{1}$ satisfies $q\left(D_{1}, E_{i}\right)=0\left(1 \leq i \leq t_{1}\right)$. Then $D_{1}$ is pseudo-effective (and effective when $D$ is effective); cf. Lemma $2.2(3)$ and [ibid. 3.3]. Let $E_{j}\left(t_{1}+1 \leq j \leq\right.$ $\left.t_{1}+t_{2}\right)$ be all prime divisors satisfying $q\left(D_{1}, E_{j}\right)<0$. Then $\left(q\left(E_{i}, E_{j}\right)\right)_{1 \leq i, j \leq t_{1}+t_{2}}$ is negative definite; cf. [ibid. 3.5] and Lemma 2.2 (7). Let $F_{2}$ be a (non-negative) combination of $E_{k}\left(1 \leq k \leq t_{1}+t_{2}\right)$ such that $D_{2}:=D-F_{2}$ satisfies $q\left(D_{2}, E_{k}\right)=0$. Then $D_{2}$ is pseudo-effective (and effective when $D$ is effective); cf. Lemma 2.2 (3) and [ibid. 3.3]. Since $X$ has finite Picard number and by Lemma 2.2 (3), this process will terminate at step $r$, and $P_{D}:=D_{r}$ and $N_{P}:=\sum_{i=1}^{r} F_{i}$ satisfy Theorem 3.1 (I); cf. [ibid. 3.7] and Lemma 2.2 (1).

Next we prove Theorem 3.1 (II). Let $D=P_{D}+N_{D}$ be as in (I). Set

$$
D_{1}:=\sigma_{1}^{*} D, \quad P_{1}:=\sigma_{1}^{*} P_{D}, \quad N_{1}:=\sigma_{1}^{*} N_{D} .
$$


Then $D_{1}=P_{1}+N_{1}$ is the Zariski $q$-decomposition for $D_{1}$, by the uniqueness in (I) and since $\sigma_{1}^{*}$ is compatible with $q(*)$ (cf. Lemma $2.2(6)$ ). Let $\pi: X_{2} \rightarrow X_{1}$ be a blowup such that the composition $\sigma_{2}=\sigma_{1} \circ \pi: X_{2} \rightarrow X$ is holomorphic. Set

$$
D_{2}:=\sigma_{2}^{*} D, \quad P_{2}:=\pi^{*} P_{1}, \quad N_{2}:=D_{2}-P_{2} .
$$

Note that $P_{2}$ is nef and $N_{2}=\sigma_{2}^{*} N_{D}+E$ for some $\sigma_{2^{-}}$(and hence $\pi$-) exceptional divisor $E$, since $\sigma_{1}$ is isomorphic in codimension one (cf. Lemma 2.2 (6)).

We claim that $E \geq 0$ and hence $N_{2} \geq 0$. Indeed, $E=\sigma_{2}^{*} D-\pi^{*} P_{1}-\sigma_{2}^{*} N_{D}$ and $-E$ is $\sigma_{2}$-nef. Furthermore, we have $\sigma_{2 *} E=0$, since $E$ is $\sigma_{2}$-exceptional. Hence, $E \geq 0$ by the negativity lemma [11, Lemma 3.39].

Now we show that $D_{k}=P_{k}+N_{k}(k=1,2)$ is the Zariski $F$-decomposition as in (II). The condition (II-i) is of course true. For a direct proof of the condition (II-iii), by the projection formula (for the first and last equalities below), since $\sigma_{1}$ is isomorphic in codimenion one, and applying (I) to $D_{1}$, for every integer $s \geq 1$, we have:

$$
H^{0}\left(X_{2}, s D_{2}\right)=H^{0}(X, s D)=H^{0}\left(X_{1}, s D_{1}\right)=H^{0}\left(X_{1}, s P_{1}\right)=H^{0}\left(X_{2}, s P_{2}\right) .
$$

To show (II-ii), we consider $D_{2}$ only (because $D_{1}$ is similar and easier), and replacing $\pi$ by a further blowup, we have only to show the assertion $(* *)$ : if $P^{\prime}:=D_{2}-F$ is nef for an effective $\mathbb{R}$-divisor $F$ then $F \geq N_{2}$. Note that $\sigma_{2 *} P^{\prime} \in \overline{\mathrm{BK}}(X)$ (cf. Lemma 2.2 $(1)(2)))$. So $\sigma_{2 *} F \geq N_{D}$ by applying Remark 3.3 to $\sigma_{2 *}\left(P^{\prime}\right)=\sigma_{2 *}\left(D_{2}-F\right)=D-$ $\sigma_{2 *} F$. Now $-\left(F-N_{2}\right)=P^{\prime}-\pi^{*} P_{1}$ is $\pi$-nef, and

$$
\sigma_{1 *} \pi_{*}\left(F-N_{2}\right)=\sigma_{2 *}\left(F-\sigma_{2}^{*} N_{D}-E\right)=\sigma_{2 *} F-N_{D} \geq 0
$$

so $\pi_{*}\left(F-N_{2}\right) \geq 0$. Hence, $F-N_{2} \geq 0$ by [ibid.]. This proves (**) and also (II-ii).

The uniqueness of the Zariski $F$-decomposition is due to the condition (II-ii). The rest of (II) is from the construction and (I). This completes the proof of Theorem 3.1.

\subsection{Proof of Corollary 3.2}

By Theorem $3.1(\mathrm{I}), D=P_{D}+N_{D}$ and $\kappa(X, D)=\kappa\left(X, P_{D}\right)$. If $q\left(P_{D}\right)>0$ then $P_{D}$ is big (cf. Lemma $\left.2.2(5)\right)$ and hence $\kappa\left(X, P_{D}\right)=\operatorname{dim} X$. Conversely, suppose that $\kappa(X, D)=\operatorname{dim} X$. Then $X$ is Moishezon and Kähler and hence projective, and $P_{D}$ (and also $D$ ) are big. So $P_{D}=A+E$ for an ample $\mathbb{R}$-divisor $A$ and an effective $\mathbb{R}$-divisor $E$. By Lemma $2.2(1), q\left(P_{D}\right) \geq q\left(P_{D}, A\right) \geq q(A, A)>0$. This proves Corollary 3.2.

\subsection{Proof of Theorem $\mathbf{1 . 2}$}

Replacing $P$ by a small multiple, we may assume that $(X, P)$ is terminal (cf. [11, Cor. 2.35]). By the minimal model program (MMP), [3, Cor. 1.4.1] and the termination of $P$-flops on hyperkähler projective manifolds (to be proved in Theorem 4.1), there is a surjective-in-codimension-one birational map $\sigma: X \rightarrow X^{\prime}$ such that $\left(X^{\prime}, P^{\prime}\right)$ (with $P^{\prime}:=\sigma_{*} P$ ) is $\mathbb{Q}$-factorial and terminal, and $K_{X^{\prime}}+P^{\prime}$ is nef. Furthermore, $\sigma=\sigma_{r} \circ \cdots \circ \sigma_{1}$, where each $\sigma_{i}: X_{i} \rightarrow X_{i+1}$ is either divisorial or a flip. Let $P_{i} \subset X_{i}$ be the image of $P$. If $\sigma_{1}: X=X_{1} \rightarrow X_{2}$ is a $\left(K_{X_{1}}+P_{1}\right)$-negative divisorial contraction with $E_{1}$ the exceptional (necessarily irreducible) divisor, then $\ell_{1}$. $\left(K_{X_{1}}+P_{1}\right)<0$ for a general curve $\ell_{1}$ in a general fibre of the restriction map $\sigma_{1} \mid E_{1}$; on the other hand, one has $\ell_{1} \cdot P_{1} \geq 0$ since $P_{1} \in \overline{\mathrm{BK}}\left(X_{1}\right) \cap \mathrm{N}^{1}\left(X_{1}\right)=\overline{\operatorname{Mov}}\left(X_{1}\right)$, the closed movable cone; see [14, Chapter III, Prop. 1.14] and Remark 3.3, or [4, Prop. 4.4, Lemma 4.9] 
and Lemma 2.2. This is a contradiction, noting that $K_{X_{1}}=0$. Thus for $i=1$, the $\sigma_{i}$ is a $\left(K_{X_{i}}+P_{i}\right)$-flip and hence a $P_{i}$-flop as defined in 4.2 , so $X_{i+1}$ is a projective hyperkähler manifold by [15, Cor. 1], and $P_{i+1} \in \overline{\mathrm{BK}}\left(X_{i+1}\right)$ by Lemma 2.2 (6); this is true for all $i$, by the same reasoning. Letting $\tau=\sigma^{-1}$, this proves Theorem 1.2.

Remark 3.7. Let $X$ be a projective hyperkähler manifold. Using Theorem 1.2, we can show that the following are equivalent.

(1) $X$ is bimeromorphic to a projective hyperkähler manifold $X^{\prime}$ with a (holomorphic) Lagrangian fibration.

(2) $X$ is bimeromorphic to a projective hyperkähler manifold $X^{\prime}$ admitting a nef, non-big $\mathbb{Q}$-divisor $L^{\prime}$ with $\kappa\left(X^{\prime}, L^{\prime}\right) \geq \operatorname{dim} X / 2$.

(3) There is a $\mathbb{Q}$-divisor $L$ with $\operatorname{dim} X / 2 \leq \kappa(X, L)<\operatorname{dim} X$.

(4) There is a dominant meromorphic map $g: X \rightarrow B^{\prime}$ with general fibre of non-general type and $\operatorname{dim} X / 2 \leq \operatorname{dim} B^{\prime}<\operatorname{dim} X$.

\section{Terminations of flops between projective hyperkähler manifolds}

In this section, we prove that any sequence of D-flops between projective hyperkähler manifolds terminates after finitely many steps.

Theorem 4.1. Let $X$ be a projective hyperkähler manifold and $D$ an effective $\mathbb{R}$ divisor on $X$. Assume that $(X, D)$ has at worst log canonical singularities. Then there exist no sequences of $D$-flops which have infinite length.

We start with the definition of $D$-flops.

Definition 4.2. Let $X$ and $X^{\prime}$ be $\mathbb{Q}$-Gorenstein normal varieties. A birational map $\phi: X \rightarrow X^{\prime}$ is said to be a $D$-flop if there exists a normal variety $Z$, projective birational morphisms $f: X \rightarrow Z$ and $f^{\prime}: X^{\prime} \rightarrow Z$ and an effective $\mathbb{R}$-Cartier divisor $D$ on $X$ which satisfy the following properties:

(1) The morphisms $f$ and $f^{\prime}$ are isomorphic in codimension one.

(2) The maps satisfy the following commutative diagram:

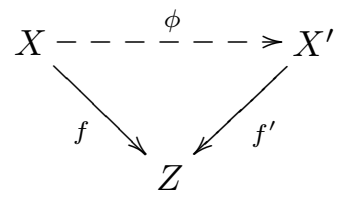

(3) The canonical divisors $K_{X}$ and $K_{X}^{\prime}$ are relatively numerically trivial.

(4) The pair $(X, D)$ has only log canonical singularities and $-D$ is $f$-ample.

(5) The proper transform $D^{\prime}$ of $D$ is $f^{\prime}$-ample.

(6) The relative Picard numbers $\rho(X / Z)$ and $\rho\left(X^{\prime} / Z\right)$ are one.

Let $\phi_{i}$ be birational maps which satisfies the following sequence:

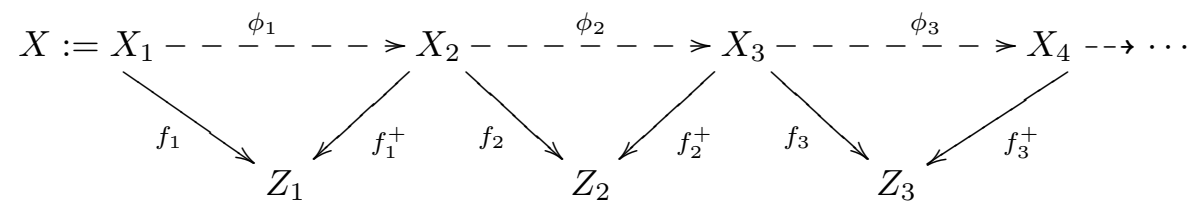


This sequence is said to be a sequence of $D$-flops if there exists an effective $\mathbb{R}$-Cartier divisor $D$ on $X$ such that $\phi_{i}$ is $D^{(i)}$-flop, where $D^{(1)}$ is $D$ and $D^{(i)}$ is the proper transform of $D^{(i-1)}$ by $\phi_{i-1}$.

Next we define log discrepancies and minimal log discrepancies.

Definition 4.3. Let $X$ be a normal variety and $D$ a Weil divisor on $X$, such that $K_{X}+D$ is $\mathbb{R}$-Cartier. For a birational morphism $\mu: X^{\prime} \rightarrow X$ from a normal variety $X^{\prime}$ and a prime Weil divisor $E^{\prime}$ on $X^{\prime}$, we define the $\log$ discrepancy $a\left(E^{\prime} ; X, D\right)$ by

$$
a\left(E^{\prime} ; X, D\right):=\left(\text { The coefficient of } E^{\prime} \text { in } K_{X^{\prime}}-\mu^{*}\left(K_{X}+D\right)\right)+1 \text {. }
$$

For a proper closed subset $W$ of $X$, we define the minimal log discrepancy of $(X, D)$ by

$$
\operatorname{mld}(W ; X, D):=\inf _{\mu\left(E^{\prime}\right) \subseteq W} a\left(E^{\prime} ; X, D\right) .
$$

Now we prove Theorem 4.1. We use the same notation as in Theorem 4.1. According to [16, Theorem], to prove Theorem 4.1, it is enough to show the following two statements.

(1) For each $i$, the function $p_{i}$ on $X_{i}$ which is defined by

$$
x \in X_{i} \mapsto \operatorname{mld}\left(x ; X_{i}, D^{(i)}\right)
$$

is lower semi-continuous.

(2) Let $\mathcal{S}$ be the set of the minimal log discrepancies defined by

$$
\mathcal{S}:=\bigcup_{i} \operatorname{mld}\left(W_{i} ; X_{i}, D^{(i)}\right)
$$

where $W_{i}$ is the exceptional locus of $f_{i}$. The set $\mathcal{S}$ satisfies the ascending chain condition.

First we prove (1). If $X$ is smooth projective and carries a holomorphic symplectic form, then $Z_{1}$ is a symplectic variety by [2, Def. 1.1]. Further, $X_{i}$ has only $\mathbb{Q}$-factorial terminal singularities, by the construction of our $D^{(i)}$-flops. Thus every $X_{i}$ is smooth by $\left[15\right.$, Cor. 1]. Then each $p_{i}$ is lower semi continuous by [5, Th. 4.4].

Next we prove (2). Since all $X_{i}$ are smooth, $\operatorname{mld}\left(W_{i}, X_{i}, D^{(i)}\right) \leq \operatorname{dim} X_{i}$. On the other hand, all pairs $\left(X_{i}, D^{(i)}\right)$ still have only log canonical singularities since each $f_{i}$ is the contraction of a $\left(K_{X_{i}}+D^{(i)}\right)$-negative extremal ray. Hence, $0 \leq \operatorname{mld}\left(W_{i} ; X_{i}, D^{(i)}\right)$. Moreover, since all $X_{i}$ are smooth and the sets of all coefficients of $D^{(i)}$ are stable, the set of $\log$ discrepancies of $\left(X_{i}, D^{(i)}\right)$ is discrete by [9, Th. 5.2]. Therefore $\mathcal{S}$ is a finite set and we are done. This proves Theorem 4.1.

Remark 4.4. If $X$ is a projective symplectic variety which has only quotient singularities, it has been announced that there exist no sequences of $D$-flops, which have infinite length in a very recent preprint [13, Cor. 1.4].

\section{Acknowledgment}

The authors would like to thank O. Fujino, M. Kawakita, N. Nakayama and H. Takagi for many suggestions, and the referee for the suggestions to improve the paper, correcting an error and encouraging us to generalize Theorem 4.1 from the dlt case to the current lc case. The first author is partially supported by Grand-in-Aid no. 18684001, Japan Society for Promortion of Sciences. The second author is partially supported by an ARF of NUS. 


\section{References}

[1] W. Barth, K. Hulek, C. Peters and A. Van de Ven, Compact complex surfaces, Springer 2004.

[2] A. Beauville, Symplectic singularities, Invent. Math., 139 (2000), 541-549.

[3] C. Birkar, P. Cascini, C.D. Hacon and J. McKernan, Existence of minimal models for varieties of log general type, J. Amer. Math. Soc. 23 (2010), 405-468.

[4] S. Boucksom, Divisorial Zariski decompositions on compact complex manifolds, Ann. Sci. école Norm. Sup. (4) 37(1) (2004), 45-76.

[5] L. Ein, M. Mustaţâ and T. Yasuda, Jet schemes, log discrepancies and inversion of adjunction, Invent. Math., 153 (2003), 519-535.

[6] T. Fujita, On Zariski problem, Proc. Japan Acad. Ser. A Math. Sci. 55(3) (1979), $106-110$.

[7] T. Fujita, Zariski decomposition and canonical rings of elliptic threefolds, J. Math. Soc. Japan 38(1) (1986), 19-37.

[8] M. Gross, D. Huybrechts and D. Joyce, Calabi-Yau manifolds and related geometries, (Lectures from the Summer School held in Nordfjordeid, June 2001), Universitext, Springer-Verlag, Berlin, 2003.

[9] M. Kawakita, Discreteness of log discrepancies over log canonical triplets on a fixed pair, J. Algebraic Geom. (to appear), also: arXiv:1204.5248

[10] Y. Kawamata, K. Matsuda and K. Matsuki, Introduction to the minimal model problem, Algebraic geometry, Sendai, 1985 (T. Oda ed.), Adv. Stud. Pure Math., 10 1987, 283-360.

[11] J. Kollár and S. Mori, Birational geometry of algebraic varieties, Cambridge Tracts in Mathematics, 134.

[12] M. Miyanishi, Noncomplete algebraic surfaces, Lecture Notes in Mathematics, 857, Springer, 1981.

[13] Y. Nakamura, On semi-continuity problems for minimal log discrepancies, arXiv:1305.1410

[14] N. Nakayama, Zariski-decomposition and abundance, MSJ Memoirs Vol. 14, Math. Soc. Japan, 2004.

[15] Y. Namikawa, On deformations of $\mathbb{Q}$-factorial symplectic varieties, J. Reine Angew. Math. 599 (2006), 97-110.

[16] V.V. Shokurov, Letters of a Bi-rationalist V, Proc. Steklov Inst. Math., 246 (2004), 315-336.

[17] O. Zariski, The theorem of Riemann-Roch for high multiples of an effective divisor on an algebraic surface, Ann. Math. (2) 76 (1962) 560-615.

Division of Mathematics, Graduate School of Science, Hokkaido University, Sapporo, 060-0810, JAPAN

E-mail address: matusita@math.sci.hokudai.ac.jp

Department of Mathematics, National University of Singapore, 10 Lower Kent Ridge ROAD, SingAPORE 119076

E-mail address: matzdq@nus.edu.sg 
